\title{
Surgical Treatment and Pathological Findings of Venous Malformations Involving a Nerve
}

\author{
Mitsunaga Narushima, MD ${ }^{1}$ Akitatsu Hayashi, MD ${ }^{1}$ Nobuyuki Kaji, MD ${ }^{1}$ Teppei Morikawa, MD \\ Ryohei Ishiura, MD ${ }^{1}$ Ai Yokoyama, MD ${ }^{3}$ Takanobu Mashiko, MD ${ }^{1}$ Takumi Yamamoto, MD ${ }^{1}$ \\ Hidehiko Yoshimatsu, MD ${ }^{1}$ Hisako Hara, MD ${ }^{1}$ Shuji Yamashita, MD ${ }^{1}$ Takuya lida, MD ${ }^{1}$ \\ Isao Koshima, MD ${ }^{1}$
}

${ }^{1}$ Department of Plastic and Reconstructive Surgery, University of Tokyo School of Medicine, Tokyo, Japan

${ }^{2}$ Department of Pathology, University of Tokyo Hospital, Tokyo, Japan

3 Department of Plastic and Reconstructive Surgery, Japanese Red

Cross Ashikaga Hospital, Ashikaga, Tochigi, Japan

J Reconstr Microsurg Open 2016;1:122-124.

\section{Introduction}

Although sclerotherapy for venous malformation (VM) is less invasive than other treatments, it is not a curative treatment and needs to be performed repeatedly over the patient's lifetime. ${ }^{1}$ In contrast, complete resection of VM can be a curative treatment, but the reconstruction after resection remains challenging from both functional and aesthetic standpoints. We herein report two cases of reconstruction using a vascularized nerve flap combined with a perforator flap after resection of VM involving a nerve.

\section{Patient Reports}

\section{Case 1}

A 10-year-old boy suffered from severe pain due to a VM on his right medial plantar area. We performed percutaneous sclerotherapy twice. While the size of the VM was diminished, the patient could not walk after sclerotherapy due to a worsening of the pain. We, therefore, planned complete resection of the $\mathrm{VM}$ and flap coverage to ease the pain without amputation.

During resection, the VM was located inside the medial plantar nerve (MPN) and strong fibrosis was noted around the nerve ( - Fig. 1A). To cover the VM defect and reconstruct the nerve defect, a $12 \times 7 \mathrm{~cm}$ superficial circumflex iliac artery perforator (SCIP) adipocutaneous flap combined with an 8-cm vascularized lateral femoral cutaneous nerve (LFCN) was elevated. The common trunk of the superficial circumflex iliac artery (SCIAc) as a pedicle of the combined flap was anastomosed to the medial plantar artery. The MPN was anastomosed to the LFCN. A histopathological examination of the resected specimen

received

May 28, 2016

accepted after revision

June 22, 2016

published online

August 8, 2016
Address for correspondence Mitsunaga Narushima, MD, Department of Plastic and Reconstructive Surgery, University of Tokyo School of Medicine, 7-3-1 Hongo, Bunkyo-ku, Tokyo, 113-0033, Japan (e-mail: sancho-ps@umin.ac.jp).

revealed numerous abnormal blood vessels with uneven thickness and dense fibrosis within and around the MPN ( - Fig. 1B).

Four years after the operation, the Semmes-Weinstein (SW) scores for the great toe, medial plantar area, and SCIP flap were observed to be $3.84,3.61$, and 4.31 , respectively.

\section{Case 2}

A 12-year-old girl suffered from an enlargement of a VM involving an ulnar nerve (UN) with increasing pain on the ulnar side of the left hand and distal part of the forearm. Due to a large volume of VM and the inclusion of the UN, we were concerned about the necessity of multiple sclerotherapy and the exacerbation of pain after sclerotherapy.

We, therefore, performed complete resection of the VM that involved the superficial branch of the UN and the skin overlaying the VM ( - Fig. 1C). The intact deep branch of the UN was preserved. A $7.5-\mathrm{cm}$ vascularized LFCN flap with a $7 \times 3 \mathrm{~cm}$ adiposal SCIP flap was taken from the left groin area. The SCIAc as a pedicle of the combined flap was anastomosed to the side wall of the ulnar artery. The LFCN was anastomosed to the superficial branch of the UN at the wrist to the common palmar digital nerve of the ring finger and the little finger. The adipose flap was wrapped around the LFCN. A histopathological examination of the resected specimen revealed that numerous abnormal blood vessels surrounded and compressed the UN. However, the fibrosis around the UN was minimal ( - Fig. 1D). At 1 year after surgery, the SW score was 2.36 for both the little finger and the ring finger.

In both cases, the patients achieved pain relief, and no recurrence was observed at 4 years and 1 year postoperatively, respectively.

Copyright $@ 2016$ by Thieme Medical Publishers, Inc., 333 Seventh Avenue, New York, NY 10001, USA. Tel: +1(212) 584-4662.
License terms

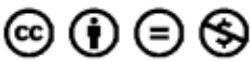

$10.1055 / \mathrm{s}-0036-1587340$ ISSN 2377-0813. 


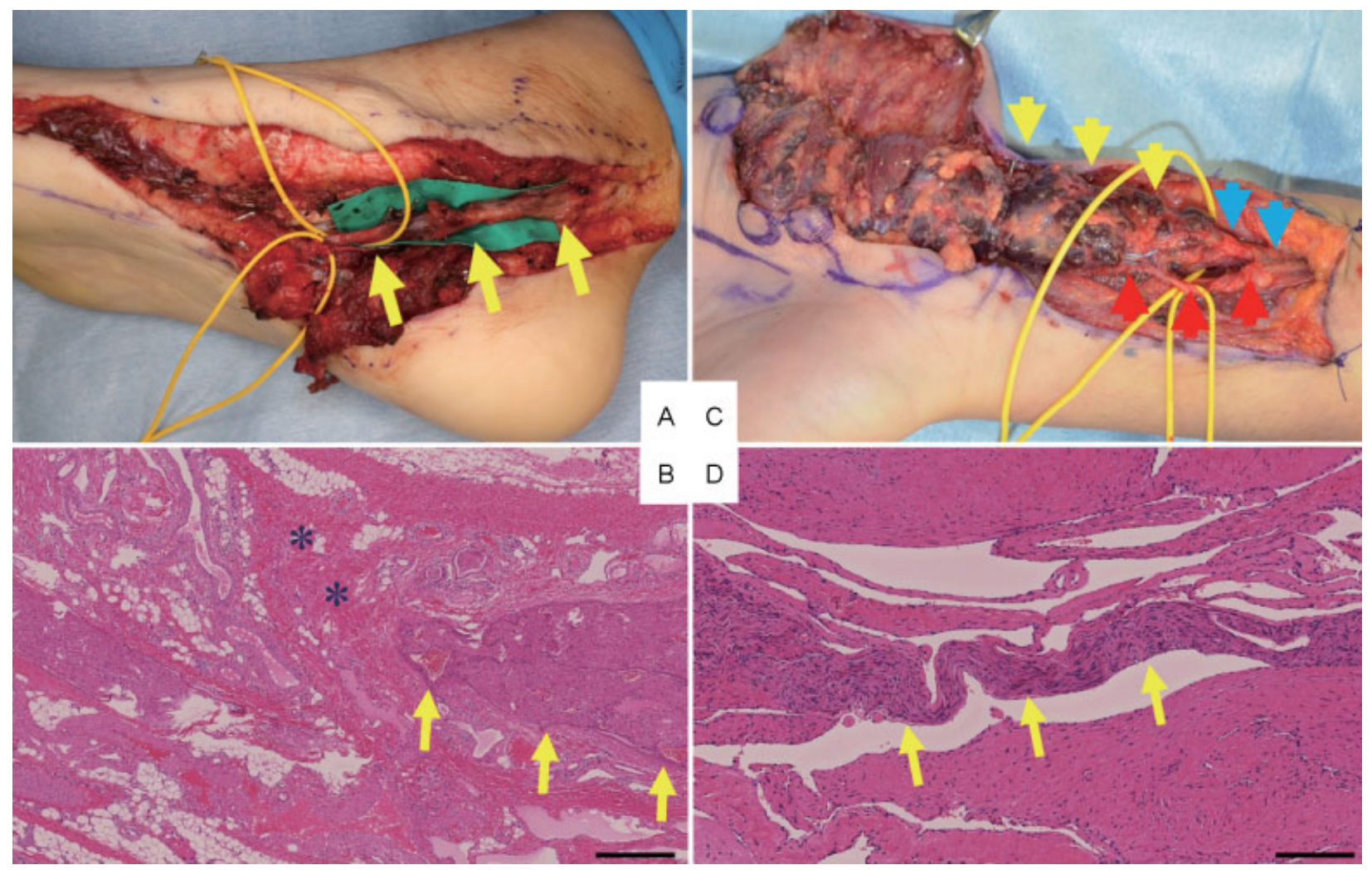

Fig. 1 (A) A venous malformation (VM) involving the right medial plantar area of a 10-year-old boy (case 1). Yellow arrow: the medial plantar nerve. (B) The histopathological findings of the resected specimen in case 1. The medial plantar nerve (yellow arrows) was surrounded by abnormal blood vessels. There was dense fibrosis within and around the medial plantar nerve (asterisks). Hematoxylin and eosin staining. Bar: 200 $\mu \mathrm{m}$. (C) A VM involving an ulnar nerve on the ulnar side of the left hand and distal part of the forearm of a 12-year-old female (case 2). Red arrow: ulnar artery. Blue arrow: intact area of the ulnar nerve. Yellow arrow: ulnar nerve including the VM. (D) The histopathological findings of the resected specimen in case 2 . The ulnar nerve (yellow arrows) was surrounded and compressed by abnormal blood vessels. In contrast to case 1 , the fibrosis around the ulnar nerve was minimal. Bar: $200 \mu \mathrm{m}$.

\section{Discussion}

Mimura et al reported that pain remained in 2 (6.9\%) out of 29 patients and worsened in 1 (3.4\%) patient after sclerotherapy, and $5(29.4 \%)$ of 17 patients who achieved complete pain relief experienced a recurrence of pain. ${ }^{2}$ These patients may need repeated sclerotherapy. Considering the pathological findings in our two cases, for a VM either inside or surrounding a nerve, multiple sclerotherapy may cause fibrotic contracture and ischemia into a nerve and may also worsen the pain, as seen in case 1 .

Furthermore, if case 2 had required an operation after several sclerotherapy sessions, we would not have been able to separate the intact deep branch from the superficial branch of the UN due to fibrosis after sclerotherapy. Given these sequelae, we believe that primary surgical resection is the treatment of choice for VM cases with nerve involvement when complete resection is possible.

We selected a vascularized LFCN rather than a nerve graft for two reasons. ${ }^{3}$ The first reason was good and rapid axon regeneration ${ }^{4}$ and the second reason was worries about the recurrence of the VM. Hurwitz and Kerber reported that arteriovenous malformations (AVMs) rapidly enlarge into active new arteriovenous shunts when extensive resection is followed by tense wound closure or skin grafts. ${ }^{5}$ Chase et al found that complete control of AVMs might rest with the replacement or addition of normal vascularized tissue to eliminate these ischemic environments. ${ }^{6}$ The recurrent mechanism of VM remains unclear. However, the replacement of VM by normal, well-vascularized nerves and tissue may decrease the risk of recurrence of VM.

\section{Conclusion}

We believe that when complete resection is possible, primary surgical resection and reconstruction with well-vascularized tissue is the treatment of choice for VM cases with nerve involvement.

Funding

None.

Conflict of Interest

None. 
124 Venous Malformation Involving a Nerve Narushima et al.

\section{References}

1 Berenguer B, Burrows PE, Zurakowski D, Mulliken JB. Sclerotherapy of craniofacial venous malformations: complications and results. Plast Reconstr Surg 1999;104(1):1-11, discussion 12-15

2 Mimura H, Fujiwara H, Hiraki T, et al. Polidocanol sclerotherapy for painful venous malformations: evaluation of safety and efficacy in pain relief. Eur Radiol 2009;19(10):2474-2480

3 Kashiwa K, Kobayashi S, Nasu W, Kuroda T, Higuchi H. Facial nerve reconstruction using a vascularized lateral femoral cutaneous nerve graft based on the superficial circumflex iliac artery system: an application of the inferolateral extension of the groin flap. J Reconstr Microsurg 2010;26(9):577-582

4 Zhu Y, Liu S, Zhou S, Yu Z, Tian Z, Zhang C, Yang W. Vascularized versus nonvascularized facial nerve grafts using a new rabbit model. Plast Reconstr Surg 2015;135(2):331e-339e

5 Hurwitz DJ, Kerber CW. Hemodynamic considerations in the treatment of arteriovenous malformations of the face and scalp. Plast Reconstr Surg 1981;67(4):421-434

6 Chase RA, Hentz VR, Apfelberg D. A dynamic myocutaneous flap for hand reconstruction. J Hand Surg Am 1980;5(6):594-599 